\title{
An in situ cell to study phase transitions in individual aerosol particles on a substrate using scanning transmission $x$-ray microspectroscopy
}

\author{
T. Huthwelker, ${ }^{1}$ V. Zelenay, ${ }^{1}$ M. Birrer ${ }^{1}$ A. Krepelova, ${ }^{1}$ J. Raabe, ${ }^{1}$ G. Tzvetkov, ${ }^{2}$ \\ M. G. C. Vernooij, ${ }^{3}$ and M. Ammann ${ }^{1}$ \\ ${ }^{1}$ Paul Scherrer Institut (PSI), 5232 Villigen PSI, Switzerland \\ ${ }^{2}$ Department of Inorganic Chemistry, University of Sofia, 1164 Sofia, Bulgaria \\ ${ }^{3}$ Environmental Mineralogy, University of Fribourg, Chemin du Musée 6, 1700 Fribourg, Switzerland
}

(Received 17 June 2010; accepted 5 September 2010; published online 17 November 2010)

\begin{abstract}
A new in situ cell to study phase transitions and chemical processes on individual aerosol particles in the x-ray transmission microscope at the PolLux beamline of the Swiss light source has been built. The cell is machined from stainless steel and aluminum components and is designed to be used in the standard mount of the microscope without need of complicated rearrangements of the microscope. The cell consists of two parts, a back part which contains connections for the gas supply, heating, cooling devices, and temperature measurement. The second part is a removable clip, which hosts the sample. This clip can be easily exchanged and brought into a sampling unit for aerosol particles. Currently, the cell can be operated at temperatures ranging from -40 to $+50{ }^{\circ} \mathrm{C}$. The function of the cell is demonstrated using two systems of submicron size: inorganic sodium bromide aerosols and soot originating from a diesel passenger car. For the sodium bromide we demonstrate how phase transitions can be studied in these systems and that $\mathrm{O} 1 \mathrm{~s}$ spectra from aqueous sodium bromide solution can be taken from submicron sized particles. For the case of soot, we demonstrate that the uptake of water onto individual soot particles can be studied.
\end{abstract}

(C) 2010 American Institute of Physics. [doi:10.1063/1.3494604]

\section{INTRODUCTION}

$\mathrm{X}$-ray microspectroscopy is a very important and versatile tool in material sciences. X-ray absorption spectra offer both chemical and structural information with a very high spatial resolution on the order of some tens of nanometers. The near edge $x$-ray absorption fine structure provides insight into the electronic structure of molecules via transitions into unoccupied orbitals, hence reflecting the chemical nature of the sample. In addition, analysis of the extended x-ray absorption fine structure in the spectra gives information about the local near ordering in the vicinity of the probed atom, an information which can be crucial for the understanding of heterogeneous chemical processes (for review see for example Refs. 1 and 2).

With the recent advances of synchrotron sources in terms of flux, brightness, and stability, microspectroscopy in the soft x-ray regime (200-1500 eV) became a feasible technique. A cornerstone for this development was the progress in micro- and nanostructuring technologies, leading to improvements of both quality and availability, of Fresnel zone plates, the key components for diffractive optics. One imaging technique is the scanning transmission $\mathrm{x}$-ray microscope (STXM), where X-rays are focused to a spot size of about 30 $\mathrm{nm}$ using a zone plate. The sample is brought into this focus and the intensity of the transmitted light is detected. With appropriate scanning techniques, high resolution images with chemical contrast can be taken. Today, STXM has matured as a standard tool in many scientific fields.

Samples of interest for STXM cover many scientific fields, including studies on magnetic phenomena (e.g., Ref.
3) or studies on nanoparticles for catalytical processes (e.g., Refs. 4 and 5). In atmospheric sciences, mixed aerosols consisting of organic matter and inorganic salts are of interest. For example, morphological and spectroscopic information derived from the nonvolatile part of collected individual atmospheric particles was the base of a source apportion study. ${ }^{6,7}$ The constituents of natural aerosols include $\mathrm{NaBr}$, which is believed to play a key role in the catalytic ozone destruction observed in the polar regions of the Earth (e.g., Ref. 8). $\mathrm{NaBr}$ is also important in biochemical engineering, as the sodium bromide dihydrate $\left(\mathrm{NaBr} \bullet 2 \mathrm{H}_{2} \mathrm{O}\right)$ can be used as buffer to control the chemical potential of water in aqueous solutions. ${ }^{9}$ Examples from atmospheric sciences include organic matter from biogenic emission and also soot particles, which originate from biomass burning and from anthropogenic sources, such as industry of cars. Also these organic particles may take up water and hence play a role in cloud formation processes.

Samples from solid state physics can often be studied under vacuum conditions. In contrast, samples in the context of biology, life science, catalysis, or environmental science may require control of both temperature and gas phase composition. Changing these parameters can introduce phase changes to the sample: For example, atmospheric aerosol particles will be solid in a dry environment, but deliquesce into a liquid state or form hydrates, once humidity or temperature pass certain coexistence lines in the phase diagram. If particles consist of a multicomponent mixture, subphases may form on the submicrometer scale. Another example for 
the need of a controlled gas phase around a sample can be found when studying heterogeneous chemical reactions and catalytic processes. To study such processes in situ, special sample environments are required.

In situ cells have been developed for studies using both hard and soft x-rays. Driven by research in catalysis, an in situ cell for the energy range from 1000 to $3500 \mathrm{eV}$ was developed. ${ }^{10,11}$ Further approaches are summarized in Refs. 12 and 13. In situ cells for use at higher energies can typically be used with a whole range of detection modes, such as transmission, electron yield, or fluorescence. Moreover, for energies higher than a few $\mathrm{keV}$, macroscopically thick samples can be used. For studies of light elements, at energies below $1000 \mathrm{eV}$, additional complications arise as both the efficiency of $\mathrm{x}$-ray fluorescence and the transmission depth decrease. Hence, only electron yield, photoelectron, or transmission spectroscopy remain as viable detection techniques. The difficulty to perform various electron spectroscopies is that the mean free path of a photoelectron is short in the presence of a gas. Cells which use total electron yield $^{14}$ and a system using a differential pumping stages were developed, which allows x-ray photoemission spectroscopy (XPS) studies at pressures as high as a few millibars. ${ }^{15-18}$

Building an in situ cell, which can be used in a STXM, faces specific challenges. The typical focal distance between sample and zone plate is less than $1 \mathrm{~mm}$ and the so-called order sorting aperture (OSA) needs to be placed between zone plate and the sample. Moreover, as light leaves the focus in a divergent cone also the detector needs to be as close as possible to the sample. Hence, an in situ cell should be thinner than $1 \mathrm{~mm}$. The penetration depth of x-rays below $1 \mathrm{keV}$ is very low. For example, at the $\mathrm{O} 1 \mathrm{~s}$ edge $(540 \mathrm{eV})$, the penetration depth of $\mathrm{x}$-rays through water is about $0.5 \mu \mathrm{m}$. Hence, thin samples, a short path in the gas phase, and a very thin window are boundary conditions when building an in situ cell for a STXM. To solve this problem Drake et al. ${ }^{19}$ used "lab on a chip techniques" and built a cell which consists of two silicon nitride windows of $100 \mathrm{~nm}$ thickness, which are about $0.8 \mathrm{~mm}$ apart from each other. This concept was further improved by de Smit et al., ${ }^{5}$ who used nanotechnology to fabricate a cell with $10 \mathrm{~nm}$ thin silicon nitride windows.

Another critical issue when using an in situ cell is related to the sample handling and the transfer of the sample into the cell. In the system used by de Smit et al., ${ }^{5}$ nanoparticles are suspended in a liquid, which is passed through the cell. After drying the cell, nanoparticles remain in the cell ready for studying the sample. In the system used by Drake et al. ${ }^{19}$ a powered sample is first brought onto an individual silicon nitride window, which is then mounted onto the cell.

To study natural or artificial aerosol particles, they must be brought onto the silicon nitride window by deposition out of a gas suspension. For artificial aerosol particles this further offers the opportunity to use aerosol technology to produce them in gas suspension and to expose them to a well defined gas environment or chemical reaction prior to deposition. Such methods have the specific advantage over applying a liquid suspension to the substrate in that less additional contamination from a solvent is brought into the sample.
Especially for particle sizes of $1 \mu \mathrm{m}$ and below, coagulation of particles into aggregates can be better controlled in gas suspension than in a colloidal solution or liquid suspension.

Here, we present a new construction for an in situ cell, which is specifically designed to study atmospheric aerosols under controlled conditions. Similarly as in previous work, the sample is sitting on a silicon nitride membrane. However, this membrane is mounted in a removable clip, which can be brought into an aerosol sample apparatus. Here, aerosol particles can be easily deposited on the membrane under controlled conditions. The clips with the samples can then be stored under controlled conditions prior to its use in the STXM. To demonstrate the applicability of the cell to study micron and submicron-sized particles relevant to atmospheric sciences, we present two types of samples. First, we consider phase transitions in $\mathrm{NaBr}$ aerosol particles. Second, we show that the technique can be used to study the uptake of small amounts of water on single, submicron sized soot particles.

\section{EXPERIMENTAL SETUP}

\section{A. STXM setup}

The STXM at the PolLux beamline has been described extensively elsewhere. ${ }^{20}$ Hence, here we recall only the principle of operation. In a STXM, a zone plate is used to focus a $\mathrm{x}$-ray beam from the size of a few $100 \mu \mathrm{m}$ in diameter to a spot size on the order of $20-40 \mathrm{~nm}$, depending on the chosen zone plate. The sample is brought into the focus of the zone plate and the intensity of the transmitted beam is detected by a photomultiplier (Hamamatsu 647P, Hamamatsu Photonics, Herrsching, Germany), ${ }^{20}$ which collects photons emitted from a phosphate.

The sample holder is mounted on a $x-y$ stage, which allows taking images by scanning the sample horizontally and vertically. This stage is driven by piezos with interferometric control of the sample position. As the zone plate focuses the beam by diffraction, the focus is a spot which is surrounded by several rings, which comprise higher orders of the diffraction pattern. To suppress this higher order pattern, and also the direct beam, a pinhole, the so-called OSA is positioned between zone plate and sample. The zone plate has a central stop to suppress the direct beam. In a STXM, the distance between focus and OSA is typically on the order of a few $100 \mu \mathrm{m}$ up to $1 \mathrm{~mm}$ depending on the beam energy.

\section{B. Cell design}

Typically, the STXM at the PolLux beamline is operated at photon energies ranging from 200 to $1000 \mathrm{eV}$. At these energies, photons penetrate matter only a few micrometers at maximum. Hence, for absorption spectroscopy measurements, both sample and window must be optically thin. The material of choice for a x-ray window is a thin silicon nitride membrane on a silicon wafer frame. Figure 1 illustrates the principle of the gas cell. Two silicon nitride windows are mounted with a distance of about $200 \mu \mathrm{m}$ to each other, and a gas passes between these two windows. The front window hosts the sample; the transmitted $\mathrm{x}$-rays can pass through the back window into the detector. The cell is equipped with a 


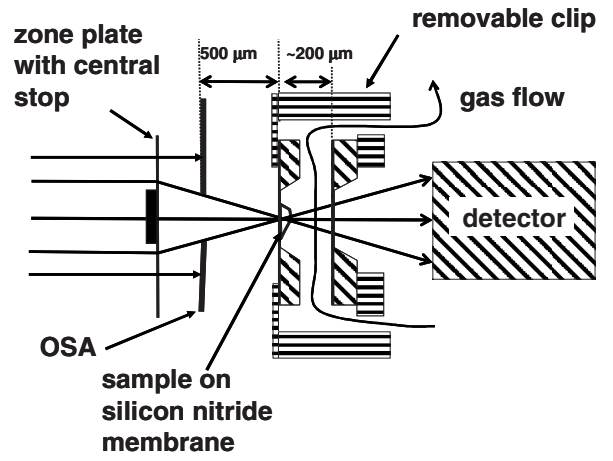

FIG. 1. Conceptual sketch of the gas cell. X-rays enter the cell from the left side and are focused by a zone plate. Both front and back windows are silicon nitride membranes $(50 \mathrm{~nm}$ thick). The front window serves as sample holder and is fixed to a removable metal clip. A Viton O-ring (see Fig. 2) ensures that the cell is gas tight.

temperature sensor and a heater. Gases are prepared by a gas mixing system outside of the microscope and are supplied to the cell via Teflon tubes. As the cell is separated gas tight from the microscope, any gases flowing through the cell cannot contaminate the microscope itself. In turn, the gas environment within the cell can be kept cleaner than in the microscope due to the significant gas turnover rate and a limited amount of materials associated with the cell and its connecting tubes.

Usually, samples for analysis in the STXM are mounted onto a thin standardized aluminum plate, which hangs in a three point mount (see Fig. 2, left panel) in the microscope. For the cell, this mounting concept is adapted. A slightly modified standard aluminum plate serves as holder for the gas cell. The cell is mounted to the plate such that the sample in the cell is in the same plane as a standard sample would be. This concept allows mounting and removing the cell easily without further complicated readjustment or rearrangements of the microscope itself. In principle, when changing between the cell and a standard sample, only a slight refo- cusing of the microscope is necessary. Figure 2 (left panel) shows the cell including all connections for electricity (temperature sensor, heater) and gas flow.

In Fig. 2 (middle panel) we detail the design of the cell. It consists of two parts: a back part, which is connected to the standard sample holder plate, and a removable clip, which serves as sample holder. The back part carries all connections for the gas flow and for the electric elements to the cell (left panel). It also hosts the back window, which is glued to the sample holder either using superglue (Loctide 406, Henkel AG, Munich, Germany) or wax (crystal bond, SPI supplier, West Chester, USA). For machining reasons, the back part is built of two parts, which are glued to each other. This part is made of aluminum because of its good heat conductivity. A hole, which is drilled into the main body of the cell, serves as channel for the gas. The gases pass through a small region between the cell body and the Viton O-ring into the cell (See red arrow in Fig. 2, left panel).

The zone plate focuses the light into a cone, which diverges behind the sample. To ensure that the exit cone is smaller than the detector size, the detector must be close to the sample. For a zone plate of $240 \mu \mathrm{m}$ in diameter with a focal length of $2 \mathrm{~mm}$ at $280 \mathrm{eV}$ and a detector diameter of $0.4 \mathrm{~mm}$, the detector must be placed at maximum $3.2 \mathrm{~mm}$ behind the focus. To allow for this short distance, a hole in the back part of the holder ensures that the detector can be moved close enough to the sample (see middle panel).

The second part of the cell is a removable clip with the silicon nitride window as sample holder (see Fig. 2). The clip is essentially a small flange sealed by a Viton O-ring. The clip is machined such that the front is a thin stainless steel membrane of $100 \mu \mathrm{m}$ thickness. On this membrane, a silicon wafer with a silicon nitride membrane is glued using a thin film of wax. The wax is chosen because it is chemically inert and because of its low vapor pressure. Moreover, by heating the clip, the silicon nitride membrane can be easily removed from the clip, which makes the clip completely re-

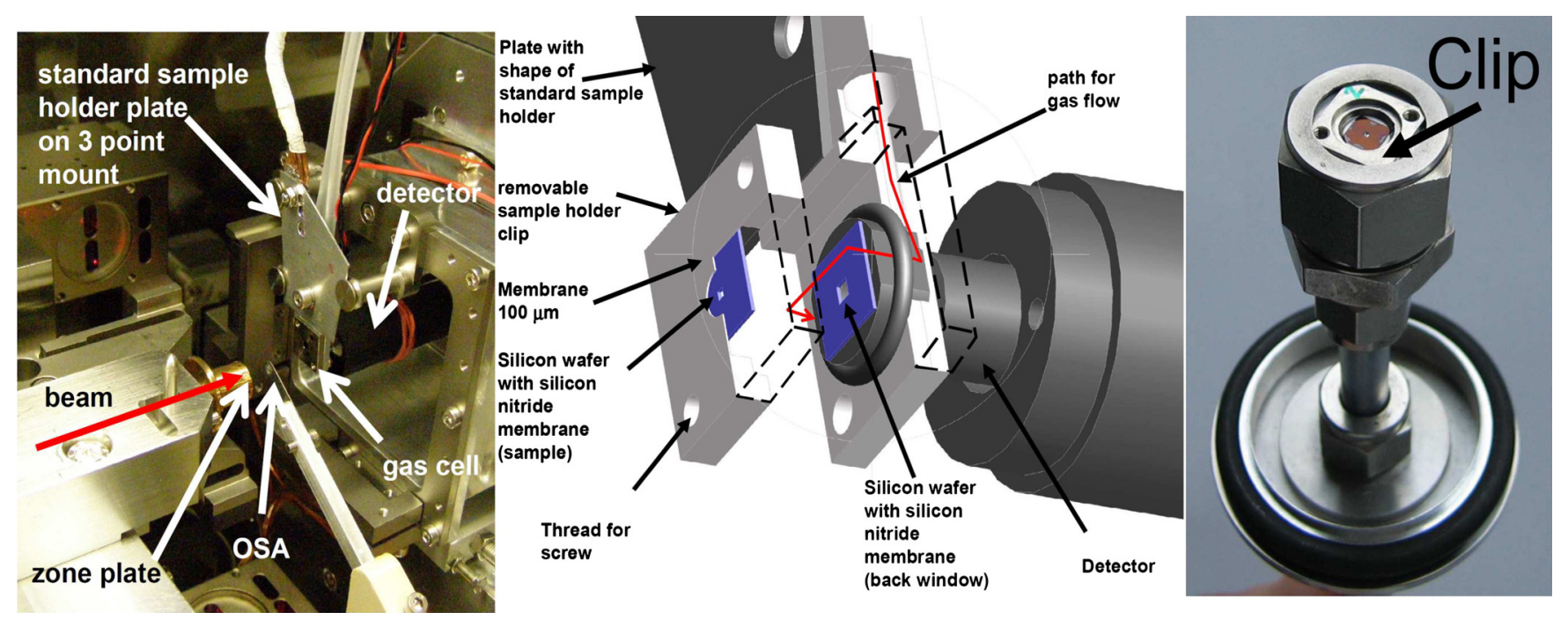

FIG. 2. (Color online) Detailed construction of the gas cell. Left panel: photo of the gas cell as built in the microscope. Cell, OSA, and detector are moved away from the focus position for better view on the elements. A removable clip hosts the sample. All connections for gases and electricity are in the back of the cell. Middle panel: three dimensional drawing of the cell. A removable clip hosts the sample. Right panel: clip mounted into equipment to collect aerosol particles. 
usable. As the wax melts at $60{ }^{\circ} \mathrm{C}$, the maximum temperature of the cell is about $40{ }^{\circ} \mathrm{C}$. However, by using a different type of glue, and a Teflon O-ring, the operating temperature of the cell can easily be increased. To load the sample onto the membrane, the whole clip can be mounted into an aerosol sampler as described below.

The dimensions of the cell have been optimized to study individual submicrometer sized aerosol particles. The x-ray transmission of the cell with and without gas phase and for different typical samples has been calculated. For all energies above the carbon edge, the overall transmission is more than $25 \%$ using windows of $50 \mathrm{~nm}$ thickness on both sides. For experiments with aqueous systems, we use water vapor buffered in helium (maximum helium pressure of 1 bar). The maximum vapor pressure of water is about 30 mbar, corresponding to about $95 \%$ relative humidity at a temperature of $25{ }^{\circ} \mathrm{C}$. This additional absorption of $\mathrm{x}$-rays by water in the gas phase is negligible. With deliquesced $\mathrm{NaBr}$ aerosol samples of $300 \mathrm{~nm}$ thickness, the overall absorption is still less than $90 \%$, ensuring that Beer's law still applies. Given the dimensions of the front window $(0.5 \times 0.5 \mathrm{~mm})$ and the back window $(1 \times 1 \mathrm{~mm})$, both of $50 \mathrm{~nm}$ thickness, the system can stand a pressure difference of about 300 mbar between the inside and the outside of the cell. For practical matters, the cell is operated at a total pressure differential of 150 mbar to minimize the stress on the windows and hence the risk of breaking them.

On the side of the cell, there is a PT100 temperature sensor to measure the temperature of the aluminum body of the cell. On the backside of the holder plate, a flexible copper braid is attached. This braid is connected to a cold reservoir, such as a cooling trap filled with liquid nitrogen. Between the cell and the attachment point of the copper braid, a small heater is mounted. The power of the heater is controlled using a commercial regulation unit (Co. ERO) which regulates the heating such that the temperature, which is measured on the cell body, remains constant. The stability of this temperature regulation is better than $0.1 \mathrm{~K}$. Temperatures as low as $-40^{\circ} \mathrm{C}$ can be established.

The gas cell can be used under a variety of conditions, ranging from a total pressure of 1 bar to pressures as low as a few tens of 1 mbar. For the experiments presented below, we built a system (see Fig. 3), which allows fast changes of the humidity in the gas cell. A gas flow of dry helium was taken directly from a helium cylinder. A second flow of dry helium was saturated by passing the gas over a water surface at a defined temperature. These two gas flows were mixed. By this procedure, we can change the humidity in the mixed gas just by changing the flow rate of the two gases. This procedure allows fast changes of the humidity in the mixed gas. The humidified gas flow can be pumped either through the cell, or via the bypass B2 directly into the pump. The latter mode is useful when mounting the cell, as one can set the gas flows to the desired values by computer control, without pumping the gas through the cell.

The total pressure is measured at the exit of the gas cell using a capacitive pressure head. The total pressure in the system is kept constant by regulating the gas flow with an electric needle valve (Pfeiffer vacuum EVR116, Asslar, Ger-

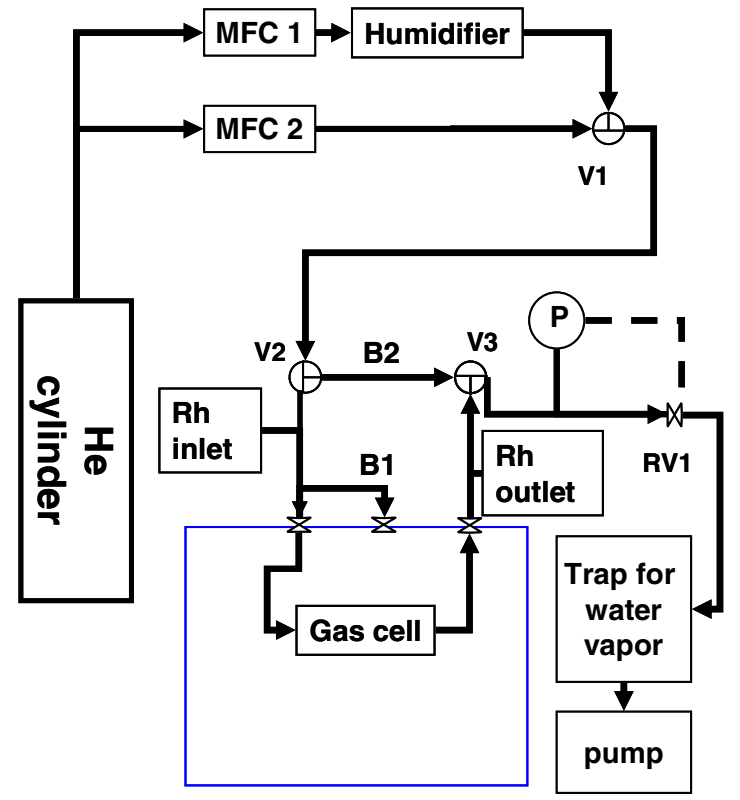

FIG. 3. (Color online) Gas flow system. MFC 1, MFC 2: mass flow controllers, V1-V3 valves, RV 1 electric needle valve, type Pfeiffer EVR 116, $\mathrm{P}$ : pressure head. B1 and B2: bypass 1 and 2, Rh inlet and Rh outlet denote capacitive humidity sensor.

many) at the exit of the system. The valve is computer controlled using a proportional-integral-derivative (PID) regulator as implemented in LABVIEW. With this method, the total pressure in the flow system is kept constant within less than $0.5 \%$. After leaving the gas cell, the humid gas is pumped through a silica gel trap to remove the humidity from the gas for protection of the exit pump.

The clip with the sample is mounted to the cell under ambient conditions, i.e., at atmospheric pressure. Once the cell is mounted into the microscope, it can be pumped down. During the evacuation the cell is pumped as well via a second bypass B1 (see Fig. 3). By this method, the pressure in the cell equals the one in the microscope, minimizing the risk to break the sensitive silicon nitride membranes. Once the working pressure of $150 \mathrm{mbar}$ or less is reached, the bypass $\mathrm{B} 1$ is closed. The total flow through the gas cell is typically 20 SCCM (SCCM denotes cubic centimeter per minute at STP). The relative humidity is measured using capacity sensors (type UFT 75-AT, MELTEC, Netphen-Deutz, Germany) both at the entry and the exit lines of the gas cell. The accuracy of the relative humidity measurement is $+-2 \%$ for $10 \%-90 \%$ rh and $<4 \%$ otherwise. The relative humidity is $100 \%$, if the partial pressure of water in the gas phase equals the water vapor pressure above pure water kept at the temperature of the gas phase. As the humidity measurements were done at room temperature, the relative humidity in the cell was calculated from the measured cell temperature. For this calculation, we use the formula for the water vapor pressure above water, as given by Goff and Gratch. ${ }^{21}$

In Fig. 4, we show the temporal evolution of the relative humidity in the cell during a typical experiment. The lower two lines show the relative humidity in the inlet and the outlet of the cell. Typically these values differ by up to $8 \%$ when the gas flows through the cell. In absence of the gas flow, i.e., when the shortcut between inlet and outlet is con- 


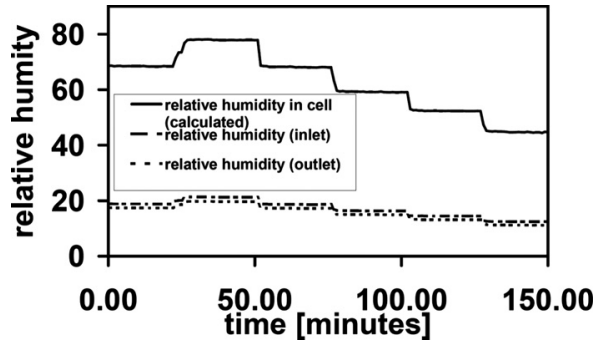

FIG. 4. Plot of measured humidity as function of time. Lower lines show the measured humidity at the inlet and outlet of the cell. The upper solid line is the humidity in the cell as calculated from the average of the humidity in the inlet and outlet (see text).

nected, the two sensors coincide within $0.1 \%$. The difference for the reading between the two sensors is due to the pressure drop over the gas cell. The length of the tubing for the inlet and outlet is equal. Hence, the pressure in the cell must be the mean value between the pressure at the inlet and the outlet. Consequently, also the humidity in the cell is given by the average of the values measured at the inlet and the outlet.

\section{Sample preparation: $\mathrm{NaBr}$ aerosol}

Aerosol particles were produced from an aqueous $\mathrm{NaBr}$ solution (10 g/l) using a homebuilt ultrasonic nebulizer and carried by a flow of nitrogen. The aerosol particles were dried by passing them through a silica gel dryer. Part of the flow is directed to a scanning mobility particle sizer, consisting of a differential mobility analyzer and a condensation particle counter to obtain the particle size distribution. Typically, we produce aerosols with diameters between $100 \mathrm{~nm}$ and $1 \mu \mathrm{m}$. These aerosol particles are charged using a radioactive krypton source providing bipolar ions, and are deposited in a static electric field $(0.8 \mathrm{keV})$ directly onto the silicon nitride membranes. The geometric configuration is such that it resembles that of an impactor assisting the inertial deposition of larger particles, while the electric field supports deposition of the smaller particles, which would otherwise escape. The deposition characteristics were not quantified in detail. However, the particle density on the substrate was checked for each sample by looking on the sample with a microscope, which also allowed determining an approximate deposition velocity to predict sampling times based on the known aerosol characteristics. Typical sampling times for a dense aerosol with about $5 \times 10^{5}$ particles per $\mathrm{cm}^{3}$ are 5-15 min. An image of the clip mounted in the sampling device is shown in Fig. 2, right panel. The unit for the sample deposition is designed to host the complete clip with the silicon nitride membrane. After deposition of the sample, the clip is stored under dry conditions and prior to the experiments mounted onto the cell.

\section{Sample preparation: Soot particles}

Soot particles were taken from the exhaust of a diesel passenger car. Right after emission from the car exhaust pipe, airborne soot particles were processed in the PSI smog chamber (for details of the smog chamber see Ref. 22). In the chamber, the soot particles suffer photochemical aging for a few hours. After this processing, the soot particles were col-

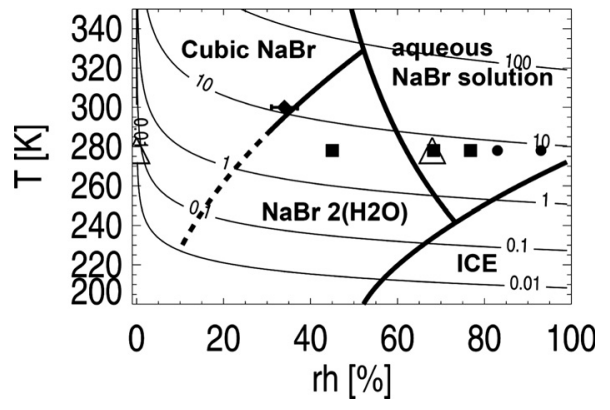

FIG. 5. NaBr Phase diagram as a function of the temperature and the relative humidity. The relative humidity refers to the vapor pressure above water at the given temperature. Stability domains for ice, sodium bromide $(\mathrm{NaBr})$, sodium bromide dihydrate $\left(\mathrm{NaBr} \cdot 2 \mathrm{H}_{2} \mathrm{O}\right)$, and an aqueous sodium bromide solution are marked (thick lines). The thin lines are isolines, which show the water partial pressure in $\mathrm{hPa}$ at given temperature and relative humidity, calculated after Ref. 21. Solid squares mark the thermodynamic conditions of the experiments presented in Fig. 7, solid circles represent the conditions for the data presented by Cappa et al. as shown in Fig. 7. Triangle: thermodynamic location of the liquid and dry sample shown in Fig. 6, upper panel, diamond with bar: thermodynamic location of hydrate formation observed in Fig. 6, lower panel.

lected onto silicon nitride membranes using the sampling unit as described above. After sampling, the samples were studied in the gas cell at room temperature under conditions of different humidity.

\section{RESULTS}

To demonstrate that the cell can be used to study phase transitions, we consider the $\mathrm{NaBr}$ water system. It is instructive to consider the $\mathrm{NaBr}$-water phase diagram, which is shown as a function of the temperature and the relative humidity in Fig. 5. In the diagram, the relative humidity refers to the vapor pressure above pure water at the given temperature $T$ : At $100 \%$ relative humidity the water vapor pressure in the system equals the saturation vapor pressure above water at temperature $T$ (or supercooled water for temperatures below $0{ }^{\circ} \mathrm{C}$ ). There are four thermodynamically stable phases: cubic sodium bromide $(\mathrm{NaBr})$, the sodium bromide dihydrate $\left(\mathrm{NaBr} \cdot 2 \mathrm{H}_{2} \mathrm{O}\right)$, the aqueous $\mathrm{NaBr}$ solution, and ice. To construct the phase diagram, coexistence lines have been derived from literature data. The water vapor pressure was calculated using the formula given by Goff and Gratch, ${ }^{21}$ which is a reasonable approximation for temperatures above $230 \mathrm{~K}^{23}$ The ice-liquid coexistence line has been calculated from the ice vapor pressure using a parameterization derived from ice vapor pressure measurements taken from the CRC handbook. ${ }^{24}$ The coexistence line between the sodium bromide dihydrate and an aqueous $\mathrm{NaBr}$ solution was calculated from the vapor pressure above saturated $\mathrm{NaBr}$ solutions. ${ }^{25}$ The coexistence line between solid $\mathrm{NaBr}$ and the solid $\mathrm{NaBr} \cdot 2 \mathrm{H}_{2} \mathrm{O}$ hydrate was taken from Halling. ${ }^{9}$ The phase diagram shows that crystalline $\mathrm{NaBr}$, the $\mathrm{NaBr} \bullet 2 \mathrm{H}_{2} \mathrm{O}$ hydrate, or an aqueous $\mathrm{NaBr}$ solution can be thermodynamically stable at room temperature, depending on the vapor pressure in the gas phase. For a relative humidity below about $\sim 35 \%$, pure $\mathrm{NaBr}$ is the thermodynamically stable phase. For a relative humidity between $35 \%$ and about $58 \%$, 

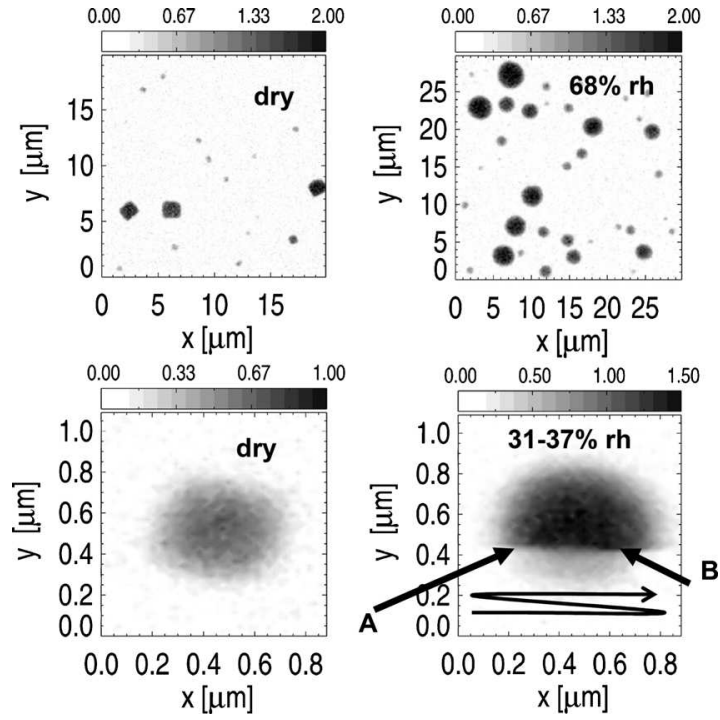

FIG. 6. Phase transitions on $\mathrm{NaBr}$ particles. Left upper panel: collected $\mathrm{NaBr}$ particles in dry helium gas flow $\left(\sim 27^{\circ} \mathrm{C}\right)$. Right upper panel: different region of the sample after several increases of the relative humidity ( $\sim 27^{\circ} \mathrm{C}$ ). Left lower panel: individual $\mathrm{NaBr}$ particle in dry environment $\left(5^{\circ} \mathrm{C}\right)$. Right lower panel: image taken during the phase transition from cubic $\mathrm{NaBr}$ to hydrate phase at about $38 \%$ rh in the cell $\left(5{ }^{\circ} \mathrm{C}\right)$. The arrow indicates the course of the scanning of the image. All data are shown in units of optical density (see color bar at top of image). Numbers on top of the image show the grayscale in units of the optical density.

the sodium bromide dihydrate is stable. Hence, phase transitions can be induced by simply changing the relative humidity in the gas phase.

\section{A. Images of individual aerosol particles during and after phase transitions}

In Fig. 6, we show STXM images of the deposited $\mathrm{NaBr}$ particles and their response to changing relative humidity in the cell. The upper left panel shows solid $\mathrm{NaBr}$ particles after deposition, which were deposited on the silicon nitride membranes using the procedure as described above. The cubic shape of the particles is apparent, indicating the presence of $\mathrm{NaBr}$ single crystals. The upper left image was taken while dry helium was passing through the cell. The upper right image shows another region once the sample was brought to a temperature of $+5{ }^{\circ} \mathrm{C}$ and a relative humidity of $68 \%$ in the cell. Now, the sample is in the stability domain of an aqueous $\mathrm{NaBr}$ solution (see triangle in Fig. 5). Clearly, a phase transition has occurred and the cubic shaped crystals have converted into round droplets.

We can determine the relative humidity when the phase transition starts by taking a series of images while slowly increasing the humidity in the cell. First, we let the particles effloresce by lowering the humidity in the gas to below $1 \%$. Then, repeated images of the same particle were taken without changing the energy, while simultaneously increasing the relative humidity in small steps. This experiment was done at a temperature of $27{ }^{\circ} \mathrm{C}$. In Fig. 6, lower left panel, the particle still shows its cubic shape, as it is typical for the $\mathrm{NaBr}$ crystal, at a relative humidity of $31 \%$. The lower right panel shows the same particle during the start of the phase transition. The image scan started in the lower left corner of the

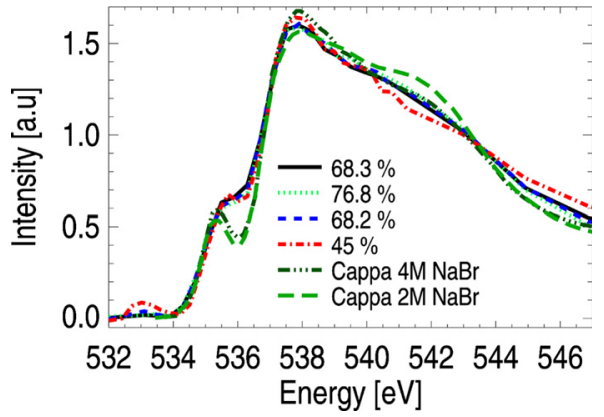

FIG. 7. (Color online) Spectra of different individual $\mathrm{NaBr}$ aerosol particles at a temperature of $5{ }^{\circ} \mathrm{C}$ in the cell. Data for $68 \%$ and $76 \%$ relative humidity are taken in the liquid stability domain (See Fig. 5). The data for $45 \% \mathrm{rh}$ are taken in the hydrate stability domain. For comparison, we show data taken from Cappa et al. (Ref. 26) taken from solutions with a concentration of $2 M \mathrm{NaBr}$ and $4 M \mathrm{NaBr}$.

image, and for all lines taken prior to the point marked as A, the particle remained unchanged. At point A the phase transition starts and has finished when the scan has reached the point marked with B. Obviously, the phase transition has started during the scan. It is not clear from the data whether the bulk of the sample or only the surface of the particle is converted to the hydrate. From the measured relative humidity prior and after the phase transition, we conclude that the transition occurred between a relative humidity of $31 \%$ and $37 \%$. For comparison, from the parameterization given by Halling, ${ }^{9}$ we calculate the phase boundary between the solid $\mathrm{NaBr}$ crystal and the sodium bromide dehydrate at a relative humidity or $32 \%$, which is in consistence with the observation. The discussion of the detailed kinetics or the question whether we observe bulk or surface processes, is beyond the scope of this paper, and subject to further study. Rather, the example shows that we can reliably create conditions where phase transitions occur. Moreover, the consistency between the thermodynamic conditions of the observed transition point and the theoretical location of the phase transition demonstrates that our method to control humidity and temperature works reliably.

\section{B. Spectra of individual aerosol particles at a temperature of $5^{\circ} \mathrm{C}$}

In Fig. 7, we show x-ray absorption spectra taken at the O1s edge of individual aerosol particles. The spectra were derived from stacks of images taken at different photon energies using the methods described in the Appendix. Each spectrum was taken from a different individual particle to minimize the effects of radiation damage to the measured spectra.

All data were taken at a temperature of $5{ }^{\circ} \mathrm{C}$. The first spectrum was taken at a relative humidity of $68.7 \%$, and then the humidity was increased to $76 \%$ and reduced back to $68 \%$ rh. These three spectra were taken in the stability domain of an aqueous $\mathrm{NaBr}$ solution (cf. Fig. 5). We note that the two spectra taken at $68 \%$ rh nicely coincide. We have also plotted spectra of a $4 \mathrm{M}$ and $2 \mathrm{M}$ solution, which were taken from Fig. 1 in Cappa et al. ${ }^{26}$ To ease comparison of the spectra measured here with the work of Cappa et al., ${ }^{26}$ we normalize all spectra to their integrated intensity in the region between 
536 and $550 \mathrm{eV}$ as described in the Appendix. In these spectra, the absorption in the region around $541.5 \mathrm{eV}$ decreases toward lower concentration, while the peak height at $538 \mathrm{eV}$ increases. The data shown here, qualitatively extend this trend, as the absorption in the region around 541.5 is even more reduced in our data, which were measured at higher concentrations of $5.9 \mathrm{M}(76 \% \mathrm{rh})$ and $6.9 \mathrm{M}$ (for $68 \% \mathrm{rh}$ ), as calculated from the water vapor pressure using the parameterization as given by Patil et al. ${ }^{27}$ We note that the energy resolution in our spectra is about $1 \mathrm{eV}$, hence we do not resolve the preedge peak at $536 \mathrm{eV}$. The choice of this lower resolution is a compromise between flux and energy resolution. However, in principle, the beamline could be run at a higher energy resolution. ${ }^{20}$

In addition, a spectrum taken at $45 \%$ rh is also shown. This spectrum is taken in the stability domain of the dihydrate; it does not show any "bump" of increased absorption in the region around $541 \mathrm{eV}$, as observed in the liquid spectra. Currently, it cannot be clearly assigned whether this spectrum is a spectrum from a strongly supersaturated sodium bromide solution or from the hydrate. The water to $\mathrm{NaBr}$ mixing ratio, roughly assessed from the postedge and preedge absorption, is between 2 and 4, close to the one of the sodium bromide dehydrate $\left(\mathrm{NaBr} \cdot 2 \mathrm{H}_{2} \mathrm{O}\right)$. Hence, it appears likely that the spectrum taken at a relative humidity of $45 \%$ is indeed a spectrum of the sodium bromide dihydrate.

\section{Water uptake on individual soot particles}

As another example to study processes on submicronsized particles, we consider photochemically aged individual soot particles from a diesel passenger car. In Fig. 8, we show high resolution images of the same soot particle taken at different relative humidities in the gas cell. Typically, soot particles from modern passenger cars have diameters on the order of a few $100 \mathrm{~nm}$ at maximum, similarly as the particle in Fig. 8. The top and middle panels in Fig. 8 show the particle exposed to a relative humidity of $30 \%$ and $86 \%$, respectively. The images are taken at an energy of $538.5 \mathrm{eV}$, where the absorption of oxygen is at its maximum. Both images are color coded in terms of optical density. Clearly, with rising humidity in the gas phase, the optical density has increased, indicating the uptake of water onto the particle.

In this experiment, images have been taken at nine different energies under dry and humid conditions from the same particle. The average optical density of each particle is calculated from all pixels within a region of $100 \mathrm{~nm}$ in diameter around the particles center. Given the beam spot size of about $30-40 \mathrm{~nm}$, the calculated average reflects a region of about $160 \mathrm{~nm}$ in diameter. The lower panel of Fig. 8 shows the difference of this average optical density between these two images. To calculate the optical density, we calculated $I_{0}$ from the histogram of the image, as described in the Appendix. The difference spectrum shown in the lower panel of Fig. 8 indicates the uptake of an oxygen containing species, which is most likely water. We note that the optical density of 0.02 would imply an uptake of 20-40 ML of water onto the particle. However, there is also a small rise in absorption at $532 \mathrm{eV}$, which may indicate either some uptake of organic matter or some chemical change to the particle due
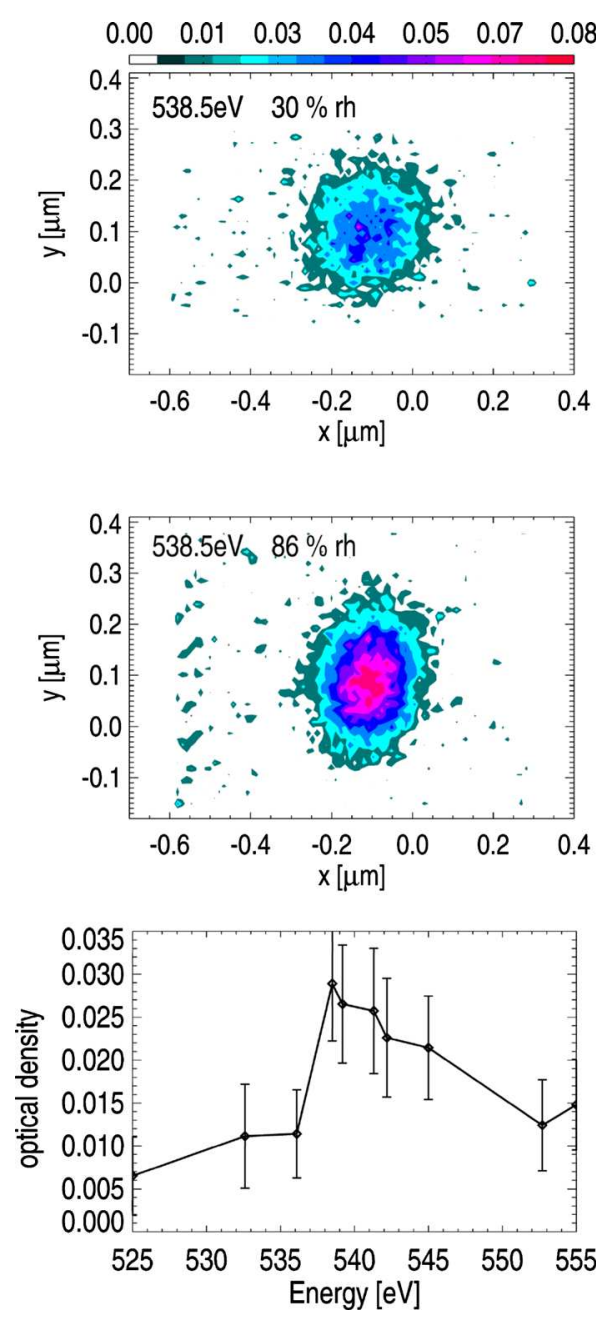

FIG. 8. (Color online) Image and spectrum of one individual soot particle, taken at different relative humidities. Top panel: $30 \%$ relative humidity, middle panel: $86 \%$ relative humidity. The color code of the images is in units of optical density. Bottom panel: spectrum calculated from the difference of the average optical density in a circle of $100 \mathrm{~nm}$ in the inner part of the particle.

to the uptake of water. Again, a complete analysis of this sample is beyond the scope of this paper, rather the example serves to illustrate the possibilities of the technique. The error bars in Fig. 8 are calculated from counting statistics, assuming an error of $N^{1 / 2}$ for $I$, sigma from the histogram width as error for $I_{0}$ and using Gaussian error propagation to estimate the error of the optical density. From this analysis, it is clear that the observed change in optical density is statistically significant.

\section{SUMMARY AND CONCLUSIONS}

We have built a gas cell for the STXM at the PolLux beamline. The gas cell is specially designed to study micronsized individual aerosol particles under controlled conditions of temperature and humidity. A removable clip with a silicon nitride membrane hosts the sample. This clip can be easily removed and replaced on the cell. This is very useful, as many clips with different samples can be prepared for a specific beam time, and, depending on the course of the experiment, exchanged fast. 
As an example for the performance, we have used $\mathrm{NaBr}$ aerosol particles. We could show that we can induce phase transitions by changing temperature and humidity in the cell. Moreover, $\mathrm{O} 1 s$ spectra from individual liquid $\mathrm{NaBr}$ particles could be taken, which are consistent with spectra generated using a liquid jet experiment. Moreover, we could take spectra in the hydrate stability domain of the sample. The liquid spectra are consistent with spectra already published but extend those to the range of higher ionic strength as prevalent in the atmosphere.

\section{ACKNOWLEDGMENTS}

We would like to thank Roberto Chirico, Dr. Josef Dommen, and Dr. André Prévôt for providing soot samples and processing them in the PSI smog chamber. We thank Dr. M. Janousch for helpful comments on the manuscript.

\section{APPENDIX: DATA ANALYSIS OF IMAGE STACKS}

Spectra were derived from a series of images of the same spot taken at different energies, the so-called energy stacks. To account for the unavoidable spatial drift when taking energy stacks, the images were aligned, using the algorithm of the stack routines of the IDL based software package AXIS $2000{ }^{28}$ After alignment, the average image was calculated from all images in the stack. For this average image, contour lines were calculated for ten different absorption levels. The regions defined by these contour lines were used to define the regions used to calculate the intensity of the incoming light $\left(I_{0}\right)$ and of the absorbed light $(I)$ for each individual images, taken at different energies. $I_{0}$ was calculated from each individual image by using all pixels in the region, where the absorption in the average image was below $20 \%$ of the maximum absorption of the average image. The intensity of the transmitted light, $I$, contains all data points which were in the region with an absorption higher than $40 \%$ of the maximum absorption observed in the average image. The choices of these threshold values were confirmed by inspecting the path of the calculated contour lines in the images. The $20 \%$ threshold for the choice of $I_{0}$ makes a path just at the outside of the sample. Moreover, when changing this level, practically no differences in the spectra were found, indicating homogeneous samples. Alternatively, we used the histogram calculated form the image to determine $I_{0}$. In each individual image, the size of the sample is small compared to the size of the region, which is used to calculate $I_{0}$. Hence, the histogram shows only one pronounced peak. Its center is a direct measure for $I_{0}$. Using these algorithms allows a fully automated calculation of spectra from an energy stack.

As the samples are not of homogeneous thickness, complications may occur in the data analysis. The transmitted intensity $I_{i}$ (given in counts $\mathrm{s}^{-1} \mathrm{~m}^{-1}$ ) of the whole sample, with thickness $d_{i}$ in the $i$ th pixel of an image is given by the equation

$$
I=I_{0} \sum_{i=0}^{N} \exp \left(-\mu d_{i}\right)
$$

This equation cannot be solved analytically with respect to the physical absorption coefficient $\mu$. Using this definition, Beer's law reads for the $i$ th pixel

$$
I_{i}=I_{0} \sum \exp \left(-\mu d_{i}\right)
$$

which can be rewritten as

$$
-\mu d_{i}=\ln \frac{I_{i}}{I_{0}}
$$

This equation allows calculating the average optical density $\mu \bar{d}$ by averaging over all pixels from the whole sample by

$$
\overline{-\mu d}=\frac{1}{N} \sum_{R O I} \ln \frac{I_{i}}{I_{0}} .
$$

For the analysis of the $\mathrm{NaBr}$ spectra (Fig. 7), we carefully corrected for any drift in the data. This drift was in the order of a few percent over the range of the full spectrum. This was done by first fitting a straight line to the postedge region $(550-560 \mathrm{eV})$ to determine the drift. Then, a straight line with the same gradient was subtracted from the spectrum. Afterward, the average intensity in the preedge region was subtracted from the spectra, making the intensity in the preedge region zero. Finally, for normalization of the intensity, the spectrum was divided by its integral in the region between 534 and $560 \mathrm{eV}$.

\footnotetext{
${ }^{1}$ J. Stöhr, NEXAFS Spectroscopy (Springer, Berlin, 1996).
}

${ }^{2}$ R. P. D. C. Konigsberger, X-Ray Absorption. Principles, Applications, Techniques of EXAFS, SEXAFS, and XANES (Wiley, Canada, 1988), Vol. 92.

${ }^{3}$ K. P. Lam, A. P. Hitchcock, M. Obst, J. R. Lawrence, G. D. W. Swerhone, G. G. Leppard, T. Tyliszczak, C. Karunakaran, J. Wang, K. Kaznatcheev, D. A. Bazylinski, and U. Lins, Chem. Geol. 270, 110 (2010).

${ }^{4}$ F. M. F. de Groot, Top. Catal. 10, 179 (2000).

${ }^{5}$ E. de Smit, I. Swart, J. F. Creemer, G. H. Hoveling, M. K. Gilles, T. Tyliszczak, P. J. Kooyman, H. W. Zandbergen, C. Morin, B. M. Weckhuysen, and F. M. F. de Groot, Nature (London) 456, 222 (2008).

${ }^{6}$ S. F. Maria, L. M. Russell, M. K. Gilles, and S. C. B. Myneni, Science 306, 1921 (2004).

${ }^{7}$ L. M. Russell, S. F. Maria, and S. C. B. Myneni, Geophys. Res. Lett. 29, 16 (2002).

${ }^{8}$ J. C. McConnell, G. S. Henderson, L. Barrie, J. Bottenheim, H. Niki, C. H. Langford, and E. M. J. Templeton, Nature (London) 355, 150 (1992).

${ }^{9}$ P. J. Halling, Biotechnol. Tech. 6, 271 (1992).

${ }^{10}$ A. M. J. van der Eerden, J. A. van Bokhoven, A. D. Smith, and D. C. Koningsberger, Rev. Sci. Instrum. 71, 3260 (2000).

${ }^{11}$ J. A. van Bokhoven, A. M. J. van der Eerden, A. D. Smith, and D. C. Koningsberger, J. Synchrotron Radiat. 6, 201 (1999).

${ }^{12}$ H. D. D. Bazin and J. Lynch, in X-Ray Absorption and Fine Structure for Catalysis and Surfaces, World Scientific series on synchrotron radiation techniques and applications, Vol. 2, edited by Y. Iwasawa (World Scientific, Singapore, 1996), p. 113.

${ }^{13}$ F. M. F. de Groot, E. de Smit, M. M. van Schooneveld, L. R. Aramburo, and B. M. Weckhuysen, ChemPhysChem 11, 951 (2010).

${ }^{14}$ A. Knop-Gericke, M. Havecker, T. Neisius, and T. Schedel-Niedrig, Nucl. Instrum. Methods Phys. Res. A 406, 311 (1998).

${ }^{15}$ W. M. Heijboer, A. A. Battiston, A. Knop-Gericke, M. Havecker, R. Mayer, H. Bluhm, R. Schlogl, B. M. Weckhuysen, D. C. Koningsberger, and F. M. F. de Groot, J. Phys. Chem. B 107, 13069 (2003).

${ }^{16}$ D. F. Ogletree, H. Bluhm, E. D. Hebenstreit, and M. Salmeron, Nucl. Instrum. Methods Phys. Res. A 601, 151 (2009).

${ }^{17}$ B. Winter, R. Weber, W. Widdra, M. Dittmar, M. Faubel, and I. V. Hertel, J. Phys. Chem. A 108, 2625 (2004).

${ }^{18}$ R. Weber, B. Winter, P. M. Schmidt, W. Widdra, I. V. Hertel, M. Dittmar, and M. Faubel, J. Phys. Chem. B 108, 4729 (2004).

${ }^{19}$ I. J. Drake, T. C. N. Liu, M. Gilles, T. Tyliszczak, A. L. D. Kilcoyne, D. K. Shuh, R. A. Mathies, and A. T. Bell, Rev. Sci. Instrum. 75, 3242 (2004).

${ }^{20}$ J. Raabe, G. Tzvetkov, U. Flechsig, M. Boge, A. Jaggi, B. Sarafimov, M. G. C. Vernooij, T. Huthwelker, H. Ade, D. Kilcoyne, T. Tyliszczak, R. H. Fink, and C. Quitmann, Rev. Sci. Instrum. 79, 113704 (2008). 
${ }^{21}$ J. A. Goff and S. Gratch, Proceedings of the 52nd Annual Meeting of the American Society of Heating and Ventilating Engineers, New York, 1946, Vol. 52, pp. 95-122.

${ }^{22}$ D. Paulsen, J. Dommen, M. Kalberer, A. S. H. Prevot, R. Richter, M. Sax, M. Steinbacher, E. Weingartner, and U. Baltensperger, Environ. Sci. Technol. 39, 2668 (2005).

${ }^{23}$ D. M. Murphy and T. Koop, Q. J. R. Meteorol. Soc. 131, 1539 (2005).

${ }^{24}$ B. P. Luo, personal communication (February 23, 2010).

${ }^{25}$ A. Wexler, in CRC Handbook of Chemistry and Physics, 91st ed. (Internet
Version 2011), edited by W. M. Haynes (CRC/Taylor and Francis, Boca Raton, FL, 2010), Vol. 90.

${ }^{26}$ C. D. Cappa, J. D. Smith, K. R. Wilson, B. M. Messer, M. K. Gilles, R. C. Cohen, and R. J. Saykally, J. Phys. Chem. B 109, 7046 (2005).

${ }^{27}$ K. R. Patil, A. D. Tripathi, G. Pathak, and S. S. Katti, J. Chem. Eng. Data 36, 225 (1991).

${ }^{28}{ }_{\text {aXis }} 2000$ - Analysis of X-ray Images and Spectra, software packed to analyze x-ray absorption spectra, see http://unicorn.mcmaster.ca/axis/ aXis2000-IDLVM.html. 